\title{
Automatic MR Brain Tumor Detection using Possibilistic C-Means and K-Means Clustering with Color Segmentation
}

\author{
Hema Rajini. N \\ Department of CSE, Annamalai University, \\ Annamalai Nagar - 608002 , \\ Tamil Nadu, India.
}

\author{
Bhavani.R \\ Department of CSE, Annamalai University, \\ Annamalai Nagar - 608002 , \\ Tamil Nadu, India.
}

\begin{abstract}
Magnetic resonance imaging is often the medical imaging method of choice when soft-tissue delineation is necessary. This paper presents a new approach for automated detection of brain tumor based on k-means and possibilistic c-means clustering with color segmentation, which separates brain tumor from healthy tissues in magnetic resonance images. The magnetic resonance feature images used for the tumor detection consist of T1-weighted and T2-weighted images for each axial slice through the head. The proposed method consists of three stages namely pre-processing, segmentation and feature extraction. In the first stage, we have suppressed the noise using image filtering. In the second stage, segmentation is computed using an unsupervised k-means and possibilistic c-means clustering algorithm with color conversion. The segmentation accuracy is obtained using the silhouette method. The experimental results show the superiority of the possibilistic c-means clustering method. In the third stage, the key features are extracted using the threshold. The application of the proposed method for tracking tumor is demonstrated to help pathologists distinguish exactly tumor size and region.
\end{abstract}

\section{Keywords}

Magnetic Resonance Imaging (MRI), K-means clustering, Segmentation, Possibilistic C-means clustering, Tumor.

\section{INTRODUCTION}

Brain tumor is a cluster of abnormal cells growing in the brain. It may affect any person at almost any age. Brain tumor effects may not be the same for each person, and they may even change from one treatment session to the next. Brain tumors can have a variety of shapes and sizes; it can appear at any location and in different image intensities. Brain tumors can be benign or malignant. Low grade Gliomas and Meningiomas [1], which are benign tumors, and Glioblastoma multiforme is a malignant tumor and represents the most common primary brain neoplasm. Benign brain tumors have a homogeneous structure and do not contain cancer cells. They may be either simply be monitored radiologically or surgically eradicated, and they seldom grow back. Malignant brain tumors have a heterogeneous structure and contain cancer cells. They can be treated by radiotherapy, chemotherapy or a combination thereof, and they are life threatening. Therefore, diagnosing the brain tumors in an appropriate time is very essential for further treatments. In recent years, neurology and basic neuroscience have been significantly advanced by imaging tools that enable in vivo monitoring of the brain. In particular, magnetic resonance imaging (MRI) [2] has proven to be a powerful and versatile brain imaging modality that allows noninvasive longitudinal and 3D assessment of tissue morphology, metabolism, physiology, and function [3]. The information MRI provides, has greatly increased the knowledge of normal and diseased anatomy for medical research, and is a critical component in diagnosis and treatment planning [4]. MR imaging is currently the method of choice for early detection of brain tumor in human brain. However, the interpretation of MRI is largely based on radiologist's opinion. According to World Health Organization (WHO), there are 126 types of different brain tumors many of which arise from structures intimately associated with the brain such as tumors of the covering membranes (meningiomas) to posterior fossa. In India, totally 80,271 people are affected by various types of tumor (2007 estimates). National Brain Tumor Foundation reported highest rate of primary malignant brain tumor occurred in Northern Europe, United States and Israel. Lowest rate was found to be in India and Philippines.

In the field of brain, MRI Gibbs et al. [5] introduced a morphological edge detection technique combined with simple region growing to segment enhancing tumors on T1 MRI data. Letteboer et al. [6], proposed an interactive segmentation method for three types of tumors: full enhancing, ring enhancing and non-enhancing. Droske et al. [7], proposed a deformable model, implemented with a level set formulation, to partition the MRI data into regions with similar image properties, based on prior intensity-based pixel likelihoods for tumor tissues. Fletcher-Heath et al. [8], proposed a combination of unsupervised classification with FCM and knowledge-based (KB) image processing for segmentation of non-enhancing tumors. Zou et al., Clark et al., and Kaus et al. have proposed methods for automatic brain tumor segmentation in MRI [9-10]. Dou et al. [11] has proposed a fuzzy information fusion framework for brain tumor segmentation using T1-weighted, T2-weighted and PD images. In contour based methods, Lefohn et al. [12] has proposed a semi-automatic method for tumor segmentation using level sets. In detecting tumor from MRI, mathematical models have been proposed in numerous works $[13,14]$ that extract necessary features from the images to characterize tumors.

The rest of this paper is organized as follows. Section 2, presents the proposed technique, utilized in this work for brain tumor detection. In this section pre-processing, segmentation, and feature extraction are presented. Section 3, presents the implementation of proposed technique. Section 4 experimentally demonstrates the performance of the proposed method. Finally, section 5 describes the conclusion of this paper.

\section{PROPOSED TECHNIQUE}

Developing an efficient diagnosing method may help physicians to diagnose tumors in an appropriate time. 
Considering the T1-weighted and T2-weighted MR images as an input data, the proposed method has three main steps, preprocessing, segmentation and feature extraction, to track the tumor objects in MR brain images. The proposed technique for automatic MR brain tumor image detection is illustrated in Fig. 1.

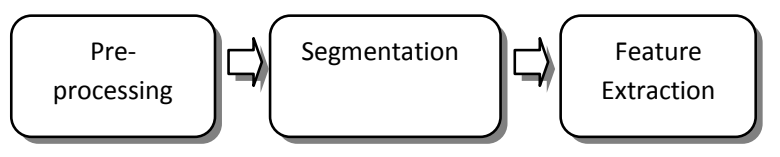

Fig 1: Methodology of the Proposed Technique

\subsection{Pre-processing}

In, the literature, there are many pre-processing techniques, which are applicable in different circumstances [15]. Moreover, in the case of inappropriate usage of these methods, the noise may be increased or small details may be eliminated [16]. The noises and artifacts on the image are reduced in pre-processing step by using a wiener filter. The general idea behind the filtering is based on statistics estimated from a local neighborhood of each pixel. By using this, the noises in the pre-processed image are reduced.

\subsection{Segmentation}

Segmentation is a major step in medical image analysis and classification for radiological evaluation or computer-aided diagnosis. One such modality that has a great deal of attention from those researching image segmentation techniques is MRI. Although MR segmentation methods have been quite successful on normal tissues, the actual methods of MR segmentation is still very much in the development stages for pathological tissues with some success recorded for specific disease processes. Image segmentation is the process of partitioning an image into a distinct region by grouping together neighborhood pixels based on some predefined similarity criterion. The similarity principle can be determined using specific properties or features of pixels representing objects in the image. So, segmentation is a pixel classification technique that allows the formation of regions of similarities in the image. When the regions are defined, features can be computed to represent regions for characterization, analysis and classification. These features will include shape and texture information of the regions as well as statistical properties, like variance and mean of gray values. In the proposed method, the segmentation accuracy of the algorithm on brain images is defined using silhouette method [17].

Image segmentation is done by clustering method. By using the proposed k-means and possibilistic c-means clustering $[18,19]$, the improved image is segmented into four classes (White Matter (WM), Gray Matter (GM), Cerebrospinal Fluid (CSF), and Abnormality). Here, by medical text book definition, the number of clusters is assumed to be four. The proposed k-means clustering is the popular clustering algorithm. In this model, the distance function is the Euclidean distance measure.

Fuzzy clustering has been shown to be advantageous over crisp clustering in that total commitment of a vector to a given class is not required in each iteration. Thus, this method is less prone to local minima. Among the various methods in fuzzy clustering, the possibilistic c-means may compensate some of the other clustering method's shortcomings [20]. The proposed clustering algorithm is the extended type of possibilistic c-means developed by Krishnapuram and Keller. In this model, the membership functions are type-II and the distance function in this method is computed based on Gustafson and Kessel algorithm [21]. In this method, the degree of fuzziness, $\mathrm{m}$, is assumed to be 2.5 .

Table 1: Normal and abnormal tissue in brain image

\begin{tabular}{ccc}
\hline \hline & T1 image & T2 image \\
\hline CSF & Dark & Dark \\
GM & Gray & Bright \\
WM & Bright & Gray \\
Abnormality & Very bright & Very bright \\
\hline \hline
\end{tabular}

Initial membership function is obtained by comparing T1weighted and T2-weighted MR images and intensity values of normal and abnormal tissue of brain image, which has been presented in table 1 . It is observed that cerebrospinal fluid (CSF) is the black area of MR image, so its initial membership function must include the black intensities. On the other hand, the abnormality is the brightest part of MRI, so its initial membership function must include the white intensities. The white matter (WM) is bright and the gray matter (GM) is gray, but their intensity levels are very similar. Therefore, their initial membership functions must include the middle intensity levels. For T2-weighted images the initial membership value is opposite of T1-weighted images. The abnormal tissue of brain in $\mathrm{T} 1$ and $\mathrm{T} 2$-weighted MR images are very bright.

Table 2 gives the segmentation accuracy of the two algorithms on brain images, where segmentation accuracy is defined using silhouette method. These silhouette average value measures the degree of confidence in the clustering assignment of a particular observation, with well clustered observations having values near 1 and poorly clustered observations having values near -1 . The silhouette width S(i) of the object $i$ is obtained using the Eq.(1).

$\mathrm{S}(\mathrm{i})=\frac{(\mathrm{b}(\mathrm{i})-\mathrm{a}(\mathrm{i}))}{\max \{\mathrm{a}(\mathrm{i}) \mathrm{b}(\mathrm{i})\}}$

For each object, we denote by the cluster to which it belongs and computes.

$a(i)=\frac{1}{|A|}-1 \sum_{j \in A, i \neq j} d(i, j)$

In the Eq.(1), a(i) is the average distance from the $\mathrm{i}^{\text {th }}$ point to the other points in its cluster and $b(i)$ is the average distance from the $i^{\text {th }}$ point to points in another cluster $\mathrm{k}$ and it is obtained using the Eq.(3).

$\mathrm{b}(\mathrm{i})=\min _{\mathrm{C} \neq \mathrm{A}} \mathrm{d}(\mathrm{i}, \mathrm{C})$

The colors are used to depict different segments in the brain This is achieved by color segmentation.

\subsubsection{K-Means Clustering Technique}

In the pixel-based classification method histogram statistics is used to define single or multiple thresholds to classify an image pixel-by-pixel. The threshold value is obtained from the analysis of the histogram of the image to classify pixels into classes. The bimodal distribution is a simple approach is to examine the histogram. The threshold can be set to the gray value corresponding to the deepest point in the histogram valley, if the histogram is bimodal. If false, the image can be partitioned into two or more regions using some heuristics 
about the properties of the image. The histogram of each partition is used to determine thresholds. By comparing the gray value of each pixel to the selected threshold, a pixel can be classified into one or the two classes. An image $f(x, y)$ can be segmented into two classes using a gray value threshold $\mathrm{T}$ then

$g(x, y)=\left\{\begin{array}{l}1 \text { if } f(x, y)>T \\ 0 \text { if } f(x, y) \leq T\end{array}\right.$

Where $\mathrm{g}(\mathrm{x}, \mathrm{y})$ is the segmented image with two classes of binary gray values " 1 " and " 0 " and $\mathrm{T}$ are the threshold selected at the valley point from the histogram.

The k-means clustering algorithm includes the following steps:

1.Select the number of clusters $\mathrm{k}$ with initial cluster centroid $\mathrm{v}_{\mathrm{i}, \mathrm{i}} \mathrm{i}=1,2, \ldots, \mathrm{k}$.

2.Partition the input data points into $\mathrm{k}$ clusters by assigning each data point $x_{j}$ to the closest cluster centroid $v_{i}$ using the selected distance measure, for example, Euclidean distance, defined as

$\operatorname{dij}=\left\|x_{j}-v i\right\|$

Where $X=\left\{x_{1}, x_{2}, \ldots x_{n}\right\}$ is the input data set.

3.Compute a cluster assignment matrix $U$ representing the partition of the data points with the binary membership value of the $\mathrm{j}^{\text {th }}$ data point to the $\mathrm{i}^{\text {th }}$ cluster such that $\mathrm{U}=$ $\left\lfloor u_{i j}\right\rfloor$, where $u_{i j} \in\{0,1\}$ for all $i, j$

$\sum_{\mathrm{i}=1}^{\mathrm{k}} \mathrm{u}_{\mathrm{ij}}=1$ for all $\mathrm{j}$ and $0<\sum_{\mathrm{j}=1}^{\mathrm{n}} \mathrm{u}_{\mathrm{ij}}<\mathrm{n}$ (6) for all $\mathrm{i}$.

4.Re-compute the centroid using the membership values by $v_{i}=\frac{\sum_{j=1}^{n} u_{i j} x_{j}}{\sum_{j=1}^{n} u_{i j}}$ (7) for all $i$.

5.If a cluster centroid or the assignment matrix does not change from the previous iteration, stop; otherwise go to step 2.

The k-means clustering method optimizes the sum-ofsquared-error-based objective function $\mathrm{Jw}_{\mathrm{w}}(\mathrm{U}, \mathrm{v})$ then

$\mathrm{J}_{\mathrm{w}}(\mathrm{U}, \mathrm{v})=\sum_{\mathrm{i}=1}^{\mathrm{k}} \sum_{\mathrm{j}=1}^{\mathrm{n}}\left\|\mathrm{x}_{\mathrm{j}}-\mathrm{v}_{\mathrm{i}}\right\|^{2}(8)$.

It can be spotted from the above algorithm that the k-means clustering method is quite sensitive to the initial cluster assignment and the choice of the distance measure. Additional criterion like within-cluster and between-cluster variances can be included in the objective function as constraints to force the algorithm to adapt the number of clusters $\mathrm{k}$, as necessary for optimization of the objective function.

\subsubsection{Possibilistic C-means (PCM) Clustering Technique}

In possibilistic c-means (PCM) approach, the clusters do not have a group of mobility, because every data point is categorized as only one cluster at a time rather than all the clusters at the same time. Consequently, an appropriate initialization is necessary for the algorithms to converge to the nearly global minimum. The advantages of PCM are that it overcomes the need to specify the number of clusters and is highly robust in a noisy environment. However, there still exist some weaknesses in the PCM, i.e., it depends highly on a good initialization and has the undesirable tendency to produce coincident clusters. Among the various methods in fuzzy clustering, the possibilistic c-means may compensate some of the other clustering method's shortcomings. The proposed clustering algorithm is the extended type of possibilistic c-means developed by Krishnapuram and Keller.

The proposed Type-II PCM model can be represented as follows:

$$
\begin{gathered}
J_{m}(X, \tilde{\mu}, c)=\min \left[\sum_{i=1}^{c} \sum_{j=1}^{N} \tilde{\mu}_{i j}^{m} D_{i j}+\sum_{i=1}^{c} \eta_{i} \sum_{j=1}^{N}\left(1-\tilde{\mu}_{i j}\right)^{m}\right](9) \\
0<\sum_{j=1}^{N} \tilde{\mu}_{i j}<N(10)
\end{gathered}
$$

S. T.: $\tilde{\mu}_{\mathrm{ij}} \in[0,1] \forall \mathrm{i}, \mathrm{j}(11)$

$\max \left(\tilde{\mu}_{\mathrm{ij}}\right)>0 \quad \forall j(12)$

Where $\mu_{\mathrm{ij}}$ is Type-II possibilistic membership values for the $\mathrm{i}^{\text {th }}$ data in the $\mathrm{j}^{\text {th }}$ cluster, and $\eta_{i}$ is positive numbers, $c$ is the number of the clusters, and $\mathrm{N}$ is the number of input data. In Eq. (9), the first term makes the distance of the data to the cluster's center be as low as possible and the second term makes the membership value in a cluster to be as large as possible.

The possibilistic c-means clustering algorithm includes the following steps:

1.Select the number of clusters $\mathrm{c}$.

2. Partition the input data points into c clusters by assigning each data point $i^{\text {th }}$ to the $\mathrm{j}^{\text {th }}$ cluster's center using the Mahalanobis distance measure

Dij $=\left\|\mathrm{x}_{\mathrm{j}}-\mathrm{v}_{\mathrm{i}}\right\|$ (13) .

3.Compute a cluster assignment matrix. The membership values for data in each cluster must lie in the interval $[0,1]$, and their sum is restricted to be smaller than the number of input data, as shown in Eqs. (10) - (12). Minimizing Eq. (9) with respect to $\mu_{\mathrm{ij}}$, leads to Eq. (14) which satisfies Eqs. (10) - (12).

$$
\tilde{\mu}_{\mathrm{ij}}=\frac{1}{1+\left(\mathrm{D}_{\mathrm{ij}} / \eta_{\mathrm{i}}\right)^{(1 / \mathrm{m}-1)}}
$$

4.As mentioned in [18], the value of $y_{i}$ determines the distance at which the membership value of a point in a cluster becomes 0.5 . In general, it is desired that $\eta_{i}$ be related to the $\mathrm{i}^{\text {th }}$ cluster and be of the order of $\mathrm{D}_{\mathrm{ij}}$ as it is shown in Eq. (15):

$\eta_{i}=K \frac{\sum_{j=1}^{\mathrm{N}} \tilde{\mu}_{\mathrm{ij}}^{\mathrm{m}} \mathrm{D}_{\mathrm{ij}}}{\sum_{\mathrm{j}=1}^{\mathrm{N}} \tilde{\mu}_{\mathrm{ij}}^{\mathrm{m}}}$

5.This method is validated using Type-II Kwon Index to recognize the structures in data and to define the number of clusters (c) and the degree of fuzziness (m). Therefore, a Type-II Kwon Index is used, which is represented by Eq. (16): $\quad \tilde{V}_{i}$ is the $\mathrm{i}^{\text {th }}$ cluster center and $\tilde{V}$ is the mean. The first term in the numerator denotes the compactness by the 
sum of square distances within clusters, and the second term denotes the separation between clusters, while the denominator denotes the minimum separation between clusters. To assess the effectiveness of clustering algorithm, the smaller the $\tilde{V}_{k}(c)$, the better the performance.

$$
\widetilde{V}_{k}(c)=\frac{\sum_{i=1}^{c} \sum_{j=1}^{N} \tilde{\mu}_{i j}^{m}\left\|x_{j}-\tilde{v}_{i}\right\|^{2}+1 / c \sum_{i=1}^{c}\left\|v_{i}-\tilde{v}\right\|^{2}}{\min \left\|\tilde{v}_{i}-\tilde{v}_{j}\right\|^{2}} i \neq j(16)
$$

The proposed Type-II PCM model, the initial membership functions, the degree of fuzziness, $\mathrm{m}$, and the number of clusters, c, must be determined. Given an input-output training pair $\left(\mathrm{x}_{\mathrm{i}}, \mu_{\mathrm{ij}}\right)$, an interval Type-II fuzzy is designed so that the error functions [22] ( $\left.\tilde{e}_{i j}\right)$ (Eq. (16)) is minimized.

$\tilde{\mathrm{e}}_{\mathrm{ij}}=\frac{1}{2}\left(\tilde{\mu}_{\mathrm{j}}\left(\mathrm{x}_{\mathrm{i}}\right)-\tilde{\mu}_{\mathrm{ij}}\right)^{2}$

where $\mu_{j}\left(\mathrm{x}_{\mathrm{i}}\right)$ is the real membership value of $\mathrm{i}^{\text {th }}$ data in $\mathrm{j}^{\text {th }}$ class and $\mu_{\mathrm{ij}}$ is the calculated membership value of $\mathrm{i}^{\text {th }}$ data in $j^{\text {th }}$ class.

\subsubsection{Color Segmentation}

The colors are used to depict different segments in the brain. In histogram-based pixel classification method for image segmentation, the gray values are partitioned into two or more clusters depending on the peaks in the histogram to obtain thresholds. The basic concept of segmentation by pixel classification can be extended to clustering the gray values of feature vector of pixels in the image. This approach is particularly useful when images with pixels representing a feature vector consisting of multiple parameters of interest are to be segmented. For example, a feature vector may consist of gray value, contrast and local texture measures for each pixel in the images. A color image may have additional color components in a specific representation such as Red, Green and Blue components in the R-G-B color coordinate system that can be added to the feature vector. The color segmentation is same as pseudo color mapping, which is defined as a method that converts colors into a single integer index that is used to select a color from a list of specified colors in a data collection. It contains a weighting vector [L1, L2, L3] for R-G-B color format and a list of R colors. An index of 1 corresponds to the first color on this list; the mapping process is as the followings:

1. Normalize the weighting vector $L$ by dividing it by its component summary.

2. Calculate $J=$ round $((\mathrm{R}-1)(\mathrm{L} 1 \times \mathrm{C} 1+\mathrm{L} 2 \times \mathrm{C} 2+\mathrm{L} 3 \times \mathrm{C} 3)$ $+1)$, where $[\mathrm{C} 1, \mathrm{C} 2, \mathrm{C} 3]=$ [red, green, blue $]$.

3. Use $J$ as an index to select one of the entries in the color list.

\subsection{Feature Extraction}

The possibilistic c-means clustering in stage two provides good initial tumor segmentation than k-means technique. The image is segmented into four classes (White Matter (WM), Gray Matter (GM), Cerebrospinal Fluid (CSF), and Abnormality). Here, by medical text book definition, the number of clusters is assumed to be four. Thresholding could provide a simple, yet effective, mechanism for separation of tumor from non-tumor pixels. The tumor shape is extracted using threshold technique. The statistical properties like area and perimeter for the tumor image region is computed.

\section{IMPLEMENTATION}

The input dataset consists of axial, T1 and T2-weighted, $256 \mathrm{x}$ 256 pixel MR brain images. The number of MR brain images in the input dataset is 50 of which 10 are of normal brain images, and 40 are of abnormal brain images.

Table 2: Segmentation accuracy for k-means and possibilistic c-means in brain image

\begin{tabular}{ccc}
\hline \hline $\begin{array}{c}\text { Clustering } \\
\text { methods }\end{array}$ & $\begin{array}{c}\text { Bilhouette } \\
\text { value }\end{array}$ & $\begin{array}{c}\text { Accuracy } \\
(\%)\end{array}$ \\
\hline $\begin{array}{c}\text { k-means } \\
\text { Possibilistic c- } \\
\text { means }\end{array}$ & 0.74 & 74 \\
\hline \hline
\end{tabular}

The abnormal brain image set consists of images of brain affected by brain lesion. The noises and artifacts on the image are reduced in pre-processing step by using a wiener filter. Image segmentation is done using k-means and possibilistic cmeans clustering, the improved image is segmented into four classes (White Matter (WM), Gray Matter (GM), Cerebrospinal Fluid (CSF), and Abnormality). We evaluate the performance of segmentation using silhouette method. Table 2 gives the segmentation accuracy of the two algorithms on brain images. The segmentation accuracy using c-means is $82 \%$ [23]. Comparing to recent results the proposed method gives better result. The tumor shape is extracted using threshold technique.

\section{RESULTS AND DISCUSSIONS}

This section portrays some experimental results on real data on brain MRI. All the input data set used for tumor detection consists of T1-weighted and T2-weighted 256x256 pixel MR brain images. The MR brain images collected from patients was acquired on 1.5 Telsa, Intera MR Scanners from Department of Radiology, Rajah Muthiah Medical College Hospital (RMMCH). The original T1 and T2-weighted MR brain image ( $256 \mathbf{x} 256$ ) which is stained with tumor as shown in Fig. 2. In the first stage, we have suppressed the noise using image filtering as shown in Fig. 3.
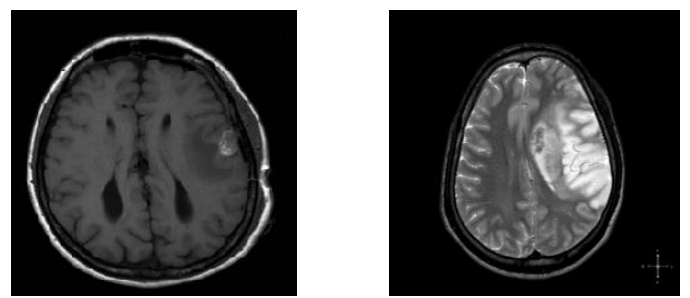

Fig 2: Original MR T1-weighted and T2-weighted brain image.
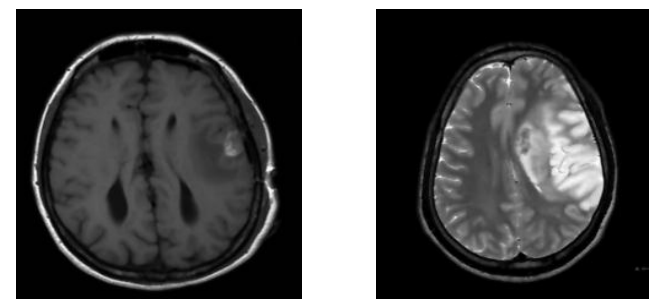

Fig 3: MR T1 and T2-weighted brain image after noise removal. 
In the second stage, segmentation is computed using an unsupervised k-means and possibilistic c-means clustering algorithm with color conversion.

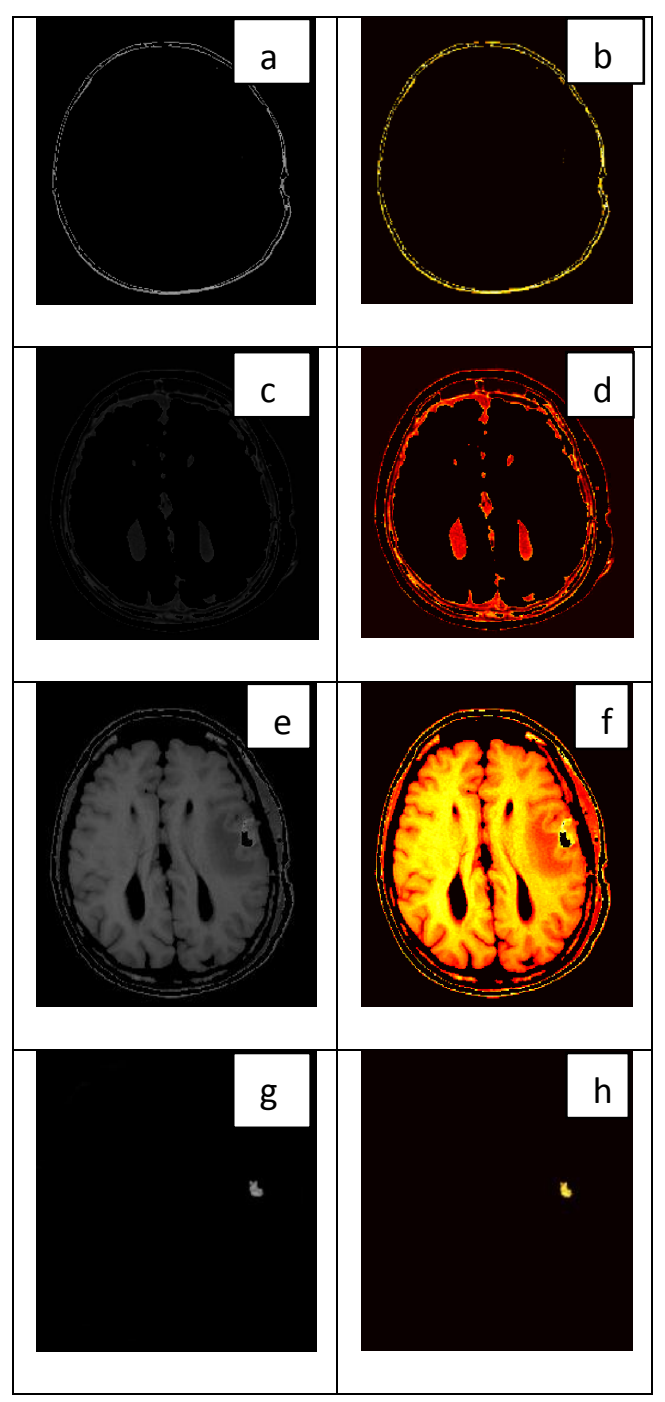

Fig 4: Color segmentation using possibilistic cmeans clustering process for $\mathrm{T} 1$-weighted image. a) Objects in cluster 1; b) Colored cluster 1; c) Objects in cluster 2; d) Colored cluster 2; e) Objects in cluster 3; f) Colored cluster 3; g) Objects in cluster 4; h) Colored cluster 4.

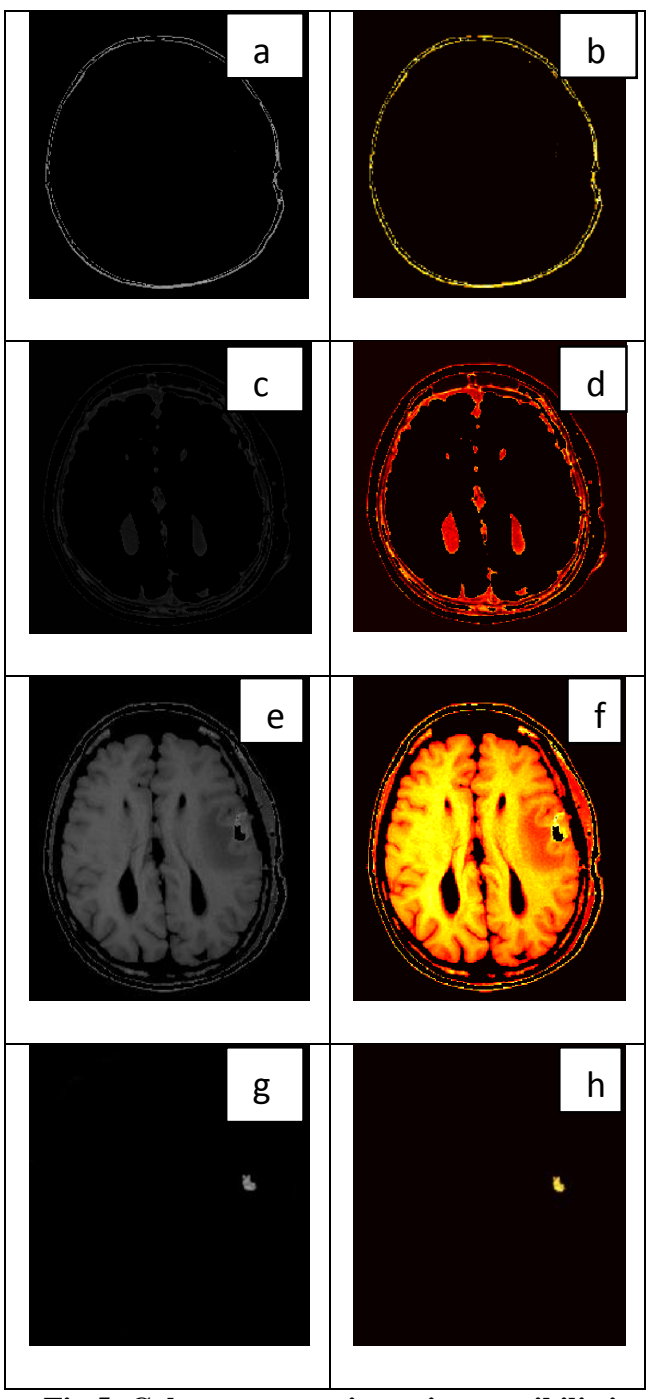

Fig 5: Color segmentation using possibilistic c-means clustering process for T2-weighted image. a) Objects in cluster 1; b) Colored cluster 1; c) Objects in cluster 2; d)

Colored cluster 2; e) Objects in cluster 3; f) Colored cluster 3; g) Objects in cluster 4; h) Colored cluster 4.

Magnetic resonance or multi-modality medical images may also require segmentation using a multidimensional feature space with multiple parameters of interest. Images can be segmented by pixel classification through clustering of all features of interest. As the image is classified into cluster classes, segmented regions are obtained by checking the neighborhood pixels for the same class label. However, clustering may produce disjoint regions with holes or regions with a single pixel. The validity of clustering results are obtained using silhouette method. The segmentation is compared with radiologist-labeled ground truth. The experimental results show that proposed possibilistic c-means clustering method achieves the better segmentation results.

The following tests show how to use color segmentation with possibilistic c-means clustering and image processing techniques to track tumor from an MR image with tumor. The possibilistic c-means technique divides the images into four tissue classes in the brain: cerebrospinal fluid, white matter, gray matter and abnormality. The number of clusters in the multi-dimensional feature space thus represents the number of classes in the image. And then labels every pixel in the image using the results from possibilistic c-means, as shown on Fig. 
4 (a-h) and Fig. 5 (a-h) shown is the image labeled by cluster index. Using pixel labels, we can separate objects in the brain image by color, which will result in four images, as shows that Fig. 4 (a-h) shown are objects in cluster 1, objects in cluster 2 , objects in cluster 3 and the final segmentation, respectively. From the final segmentation result shown, the brain regions related to the white matter, cerebrospinal fluid, gray matter and a tumor can be seen in the segmented image obtained using color segmentation with possibilistic c-means clustering.
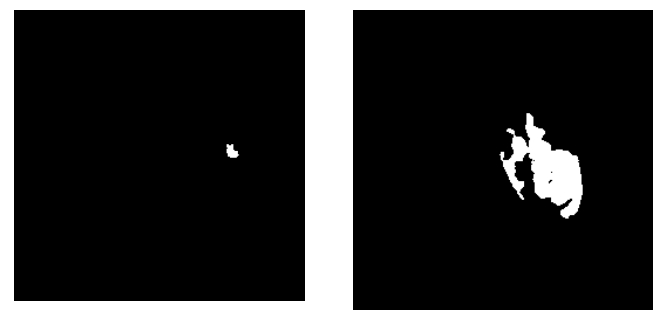

Fig 6: Tumor object of the MR brain in T1 and T2weighted image.

In the third stage, the key features are extracted using thresholding. Deducing the result and having an accurate diagnosis needs to extract the key features. Thresholding could provide a simple, yet effective, mechanism for separation of tumor from non-tumor pixels. The tumor shape is extracted using threshold technique. The statistical properties like area and perimeter for the tumor image region is computed. The tumor object of the MR brain image is shown in Fig. 6.

\section{CONCLUSION}

We have developed an automated method for the detection of tumors in brain MR images using color segmentation with kmeans, possibilistic c-means clustering and image processing techniques. The segmentation accuracy is obtained using the shilloute method. The experimental results show the superiority of the possibilistic c-means clustering method. The proposed system can help the physicians to better diagnosis human brain tumors, for further treatment. Our system has been successfully tested on large tumor causing brain tumor. The brain regions related to a tumor can be exactly separated from the brain image. This proposed method will be able to help pathologists distinguish exactly tumor size and region. This method can be further extended to segment other types of images (PET, MRS, and CT) with few modifications.

\section{REFERENCES}

[1] Ricci, P. E. and Dungan, D. H. 2001. Imaging of low and intermediate-grade gliomas. SEMRADONC, 11(2), 103112.

[2] Armstrong, T. S., Cohen, M. Z., Weinbrg, J. and Gilbert, M. R. 2004. Imaging techniques in neuro oncology. SEMONCNUR, 20(4): 231-239.

[3] Prasad, P. V. 2006. MRI: Methods and Biologic Applications, Humana Press Inc.

[4] Archibald, R., Chen, K., Gelb, A. and Renaut, R. 2003. Improving tissue segmentation of human brain MRI through pre-processing by the Gegenbauer reconstruction method. Neuroimage 20: 489-502.

[5] Gibbs, P, Buckley, D. L, and Blackband, S. J. 1996. Tumour volume determination from MR images by morphological segmentation. Phys Med Biol, 41(11): 2437-2446.

[6] Letteboer, M. M. J, Olsen, O. F and Dam, E. B. 2004. Segmentation of tumors in magnetic resonance brain images using an interactive multiscale watershed algorithm. Acad Radiol, 11: 1125-1138.

[7] Droske, M, Meyer, B and Rumpf, M. 2005. An adaptive level set method for interactive segmentation of in tracranial tumors. Neuro Res, 27(4): 363-370.

[8] Fletcher-Heath, L. M, Hall, L. O and Goldgof, D. B. 2001. Automatic segmentation of non-enhancing brain tumors in magnetic resonance images. Artif Intell Med, 21(1-3): 43-63.

[9] Zou, K. H, Wells, W. M and Kikinis, R. 2004. Three validation metrics for automated probabilistic image segmentation of brain tumours. Stat Med, 23(8): 12591282.

[10] Clark, M. C, Lawrence, L. O, Golgof, D. B, Velthuizen, R, Murtagh, F. R and Silbiger, M. S. 1998. Automatic tumor segmentation using knowledge-based techniques," IEEE Transactions on Medical Imaging, 17(2): 187-201.

[11] Dou, W, Ruan, S, Chen, Y, Bloyet, D and Constans, J. M. 2007. A framework of fuzzy information fusion for segmentation of brain tumor tissues on MR images. Image and Vision Computing, 25: 164-171.

[12] Lefohn, A, Cates, J and Hitaker, R. 2003. Interactive, GPU-based level sets for 3D brain tumor segmentation. Technical Report, University of Utah.

[13] Zook, J and Iftekharuddin, K. M. 2005. Statistical analysis of fractal-based techniques in brain tumor detection. Magn. Reson. Imaging, 23(5): 671-678.

[14] Anirban, M and Ujjwal, M. 2011. A multiobjective approach to MR brain image segmentation. Applied Soft Computing, 11: 872-880.

[15] Cheng, H. D, Shan, J, Ju, W, Guo, Y and Zhang, L. 2010. Automated breast cancer detection and classification using ultrasound images: A survey. Pattern Recognition, 43: 299-317.

[16] Bankman, I. N. 2000. Hand Book of Medical Imaging. John Hopkins University.

[17] Kannan, S. R. 2008. A new segmentation system for brain MR images based on fuzzy techniques. Applied Soft Computing, 8(4): 1599-1606.

[18] Fazel Zarandi, M. H, Zarinbal, M and Turksen, I. B. 2009. Type-II possibilistic C-mean clustering. IFSAUSEFLAT, 30-35.

[19] Vellingiri, J and Chenthur Pandian, S. 2011. Fuzzy possibilistic c-means algorithm for clustering on web usage mining to predict the user behaviour. European Journal of Scientific Research, 58(2): 222-230.

[20] Krishnapuram, R and Keller, J. M. 1993. A possibilistic approach to clustering. IEEE Transactions on Fuzzy Systems, 1: 98-110.

[21] Gustafson, D. E and Kessel, W. C. 1978. Fuzzy clustering with a fuzzy covariance matrix. Proceedings of the 17th Symposium on Adaptive Processes, 17: 761766. 
[22] Liang, Q and Mendel, J. M. 2000. Interval Type-2 fuzzy logic systems: theory and design. IEEE Transactions on Fuzzy Systems, 8: 535-550.

[23] Kannan S.R, Ramathilagam. S, Sathya.A, Pandiyarajan. R. 2010. Effective fuzzy c-means based kernel function in segmenting medical images. Computers in Biology and Medicine, 40: 572-579.

\section{AUTHORS PROFILE}

N. Hema Rajini is working with the Department of Computer Science and Engineering, Faculty of Engineering and Technology, Annamalai University.
Dr. R. Bhavani received her B.E degree in Computer Science and Engineering in the year 1989 and the M.E degree in Computer Science and Engineering in the year 1992 from Regional Engineering College, Trichy. She received her Ph.D degree in Computer Science and Engineering from Annamalai University, Chidambaram, in the year 2007. She has worked in Mookambigai college of Engineering, Keeranur from 1990 to 1994 , and She has contributed 25 technical papers in various journals and conferences. Her research interest includes Image processing, Image Segmentation, Image Compression, Image Classification, Steganography, Pattern Classification, Medical Imaging, Content Based Image Retrieval and Software metrics. 